\title{
Quality of Service and Predictability in DBMS
}

\author{
Kai-Uwe Sattler \\ TU IImenau \\ Ilmenau, Germany \\ kus@tu-ilmenau.de
}

\author{
Wolfgang Lehner \\ TU Dresden \\ Dresden, Germany \\ wolfgang.lehner@tu-dresden.de
}

\begin{abstract}
DBMS are a ubiquitous building block of the software stack in many complex applications. Middleware technologies, application servers and mapping approaches hide the core database technologies just like power, networking infrastructure and operating system services. Furthermore, many enterprise-critical applications demand a certain degree of quality of service (QoS) or guarantees, e.g. wrt. response time, transaction throughput, latency but also completeness or more generally quality of results. Examples of such applications are billing systems in telecommunication, where each telephone call has to be monitored and registered in a database, Ecommerce applications where orders have to be accepted even in times of heavy load and the waiting time of customers should not exceed a few seconds, ERP systems processing a large number of transactions in parallel, or systems for processing streaming or sensor data in realtime, e.g. in process automation of traffic control. As part of complex multilevel software stack, database systems have to share or contribute to these QoS requirements, which means that
\end{abstract}

\section{ABOUT THE AUTHORS}

Kai-Uwe Sattler (TU Ilmenau, Germany). Kai-Uwe Sattler is full professor and heads the Database and Information Systems group at the Faculty of Computer Science and Automation of the Technical University of Ilmenau, Ger-

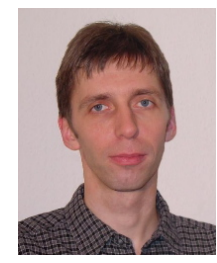
many. He received his Diploma (M.Sc.) in Computer Science from the University of Magdeburg, Germany. He received his Ph.D. in Computer Science in 1998 and his Habilitation (venia legendi) in Computer Science in 2003 from the same university.

In October 2003 he joined the Department of Computer Science and Automation of the TU Ilmenau as Professor. He has published five textbooks and more than 80 research papers. His current research interests include autonomic fea-

Permission to make digital or hard copies of all or part of this work for personal or classroom use is granted without fee provided that copies are not made or distributed for profit or commercial advantage and that copies bear this notice and the full citation on the first page. To copy otherwise, to republish, to post on servers or to redistribute to lists, requires prior specific permission and/or a fee.

EDBT '08, March 25-30, 2008, Nantes, France.

Copyright 2008 ACM 978-1-59593-926-5/08/0003 ...\$5.00 guarantees have to be given by the DBMS, too, and that the processing of database requests is predictable. Todays mainstream DBMS typically follow a best effort approach: requests are processed as fast as possible without any guarantees: the optimization goal of query optimizers and tuning approaches is rather to minimize resource consumption instead of just fulfilling given service level agreements. However, motivated by the situation described above there is an emerging need for database services providing guarantees or simply behave in a predictable manner and at the same time interact with other components of the software stack in order to fulfill the requirements. This is also driven by the paradigm of service-oriented architectures widely discussed in industry. Currently, this is addressed only by very specialized solutions. Nevertheless, database researchers have developed several techniques contributing to the goal of QoSaware database systems. The purpose of the tutorial is to introduce database researchers and practitioners to the scope, the challenges and the available techniques to the problem of predictability and QoS agreements in DBMS.

tures in database systems and large-scale distributed data management.

\footnotetext{
Wolfgang Lehner (TU Dresden, Germany). Wolfgang Lehner is full professor and head of the database technology group at the Dresden University of Technology (Technische Universität Dresden), Germany. He received his Master's degree in Computer Science in 1995 from University of Erlangen-Nuremberg.

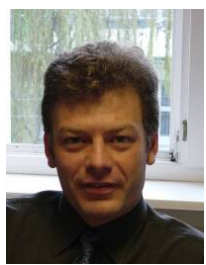

He continued his studies as research assistant at the database system group until 1998, when he earned his $\mathrm{Ph} . \mathrm{D}$. degree (Dr.-Ing.) with a dissertation on the optimization of aggregate processing in multidimensional database systems. Since 10/2002, Prof. Lehner is conducting his research, teaching his students, and is involved in multiple industrial projects at the TU Dresden. Wolfgang Lehner was also visiting scientist at IBM Almaden Research and at Microsoft Research. Up to now, Wolfgang Lehner published four text books, more than 80 reviewed research papers, and is editor of journals and four other books. His current research interests are clustered around the issue of bringing database methods into the world of service-oriented systems. He is also involved in the most prestigious nation-wide German research project on Next Generation Internet platforms (Theseus), where he contributed concepts on 'data management as a service'.
} 